\title{
Monte Carlo calculations of epithermal and fast neutron dose in a human head model for Boron Neutron Capture Therapy
}

\author{
Institute of Atomic Energy, 05-400 Otwock-Swierk \\ e-mail: kasia.tyminska@gmail.com
}

\begin{abstract}
Boron Neutron Capture Therapy is a very promising form of cancer therapy, consisting in irradiating a stable isotope of boron $\left({ }^{10} \mathrm{~B}\right)$ concentrated in tumor cells with a low energy neutron beam. This technique makes it possible to destroy tumor cells, leaving healthy tissues practically unaffected. In order to carry out the therapy in the proper way, the proper range of the neutron beam energy has to be chosen.

In this paper we continue the earlier started calculations of the optimum energy range for BNCT, taking into account the absorbed dose from fast neutrons.
\end{abstract}

Key words: BNCT, Boron Neutron Capture Therapy.

\section{Introduction}

BNCT (Boron Neutron Capture Therapy) is a form of radiotherapy that held promise of being beneficial to cancer patients with glioma, a type of brain cancer. This type of tumor is quite resistant to other treatment modalities and the prospects of its cure are very poor.

An ideal therapy for cancer would be one whereby all tumor cells were selectively destroyed without damaging normal tissues.

BNCT brings together two components that, when kept separate, have only minor effects on cells. The first component is a stable isotope of boron (boron-10) that can be 
concentrated in tumor cells by being attached to tumor-seeking compounds. The second one is a beam of low-energy neutrons. Boron-10 in or adjacent to tumor cells disintegrates after capturing a neutron. The high-energy, heavy charged particles, produced in the nuclear reaction, destroy only the cells in close proximity to the vertex, which are primarily cancer cells, leaving adjacent normal cells largely unaffected.

The dose absorbed by the tissue in BNCT consists of four components:

1. The gamma ray dose from the gamma ray component of the beam and the gamma rays induced in the tissue, mainly due to ${ }^{1} \mathrm{H}(\mathrm{n}, \gamma)^{2} \mathrm{H}$ reactions emitting $2.2 \mathrm{MeV}$ gamma rays.

2. The fast neutron dose epithermal and fast neutrons that cause 'knock-out' recoil protons from hydrogen in tissue.

3. The nitrogen dose from ${ }^{14} \mathrm{~N}$ absorbing a thermal neutron and emitting a $0.6 \mathrm{MeV}$ proton in ${ }^{14} \mathrm{~N}(\mathrm{n}, \mathrm{p}){ }^{14} \mathrm{C}$ reaction.

4. The boron dose from ${ }^{10} \mathrm{~B}$ absorbing a thermal neutron in a $\left.{ }^{10} \mathrm{~B}(\mathrm{n}, \alpha)\right)^{7} \mathrm{Li}$ reaction. The emitted $\alpha$ particle and the recoil ion ${ }^{7} \mathrm{Li}$ result in locally deposited energy, of about $2.34 \mathrm{MeV}$ in average. In $94 \%$ of the reactions the ${ }^{7} \mathrm{Li}$ ion is produced in an excited state and de-excites emitting a $477 \mathrm{keV}$ gamma ray. In the remaining $6 \%$ of events, ${ }^{7} \mathrm{Li}$ ion is emitted in the ground state.

In our previous publication [1] we have calculated selected dose components in a human head model using MCNP code. The components taken into account were:

- the gamma ray dose component,

- the nitrogen dose component,

- the boron dose component.

The calculations were made for monoenergetic neutron beams, in the energy range between $1 \mathrm{eV}$ and $1 \mathrm{MeV}$, in order to choose the optimum energy range for BNCT, where the therapeutic to the injuring dose ratio is the highest.

The results obtained for the components above mentioned dose component didn't let us choose the optimal energy range, due to the similar dependence of cross-section on energy for ${ }^{14} \mathrm{~N}(\mathrm{n}, \mathrm{p}){ }^{14} \mathrm{C}$ (the main component of the injuring dose) and ${ }^{10} \mathrm{~B}(\mathrm{n}, \alpha)^{7} \mathrm{Li}$ (therapeutic) reactions. For more complete picture we took into account the dose component coming from epithermal and fast neutrons generating 'knock-out' recoil protons from hydrogen in tissue. 


\section{Method}

The model of the head was defined within the code as a $20 \mathrm{~cm}$ diameter sphere of tissue-like material (ICRU brain) consisting of hydrogen (10.7\%), carbon (14.5\%), nitrogen $(2.2 \%)$, oxygen $(71.2 \%)$, sodium $(0.2 \%)$, phosphorus $(0.4 \%)$, sulfur $(0.2 \%)$,

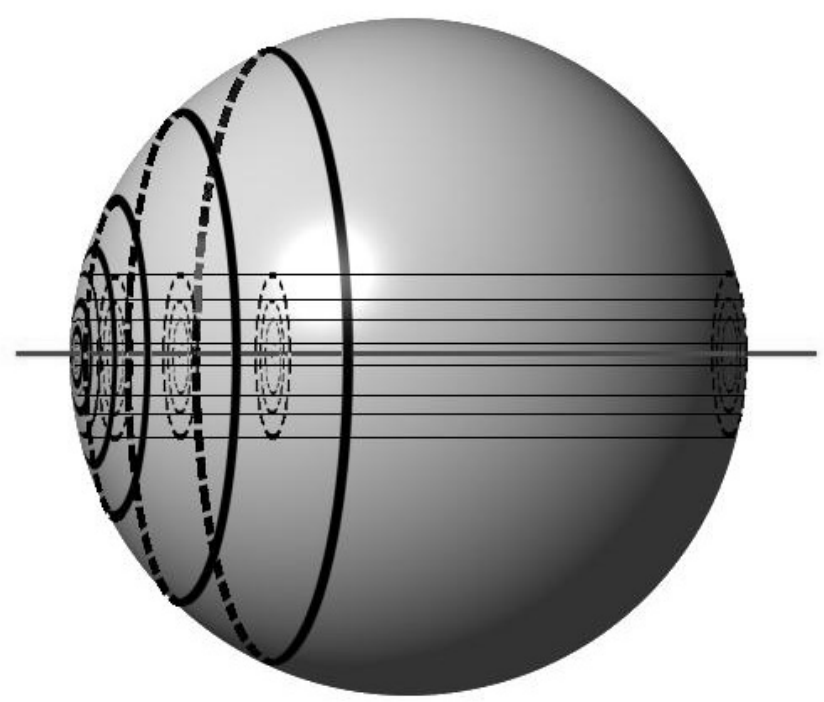

Figure 1. The model of the head used for calculations. The central line indicates the beam axis

chlorine $(0.3 \%)$ and potassium $(0.3 \%)$ of the density of $1.04 \mathrm{~g} / \mathrm{cm}^{3}$. The sphere was divided into 60 pieces of various size: 6 layers of concentric, cylindrical cells up to the depth of $7 \mathrm{~cm}$, and the rest of the head was defined as one cell (Figure 1).

\section{Results and discussion}

Figures $2 \mathrm{a}, 2 \mathrm{~b}, 2 \mathrm{c}$ present the dependence of the fast neutron absorbed dose on the depth and distance from the beam axis, for chosen energy values. The value was obtained using the neutron flux in each of the cells and multiplying it by the kerma coefficients [2], 


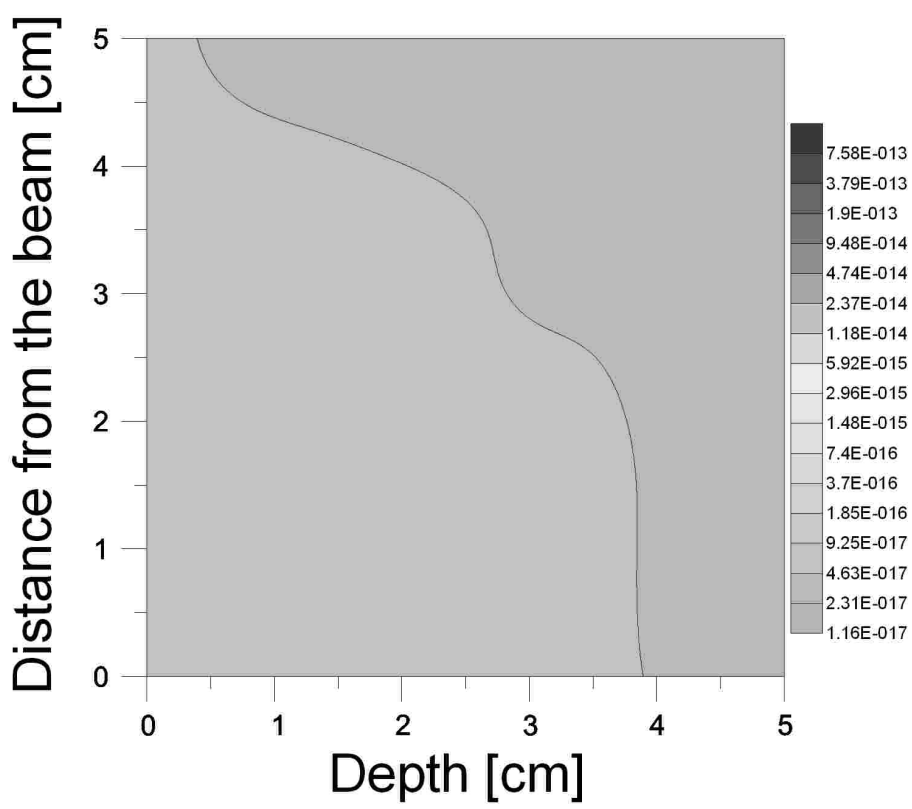

Figure 2a. The dependence of the fast neutron dose [Gy/n] on the depth and the distance from the beam for $1 \mathrm{eV}$ beam energy

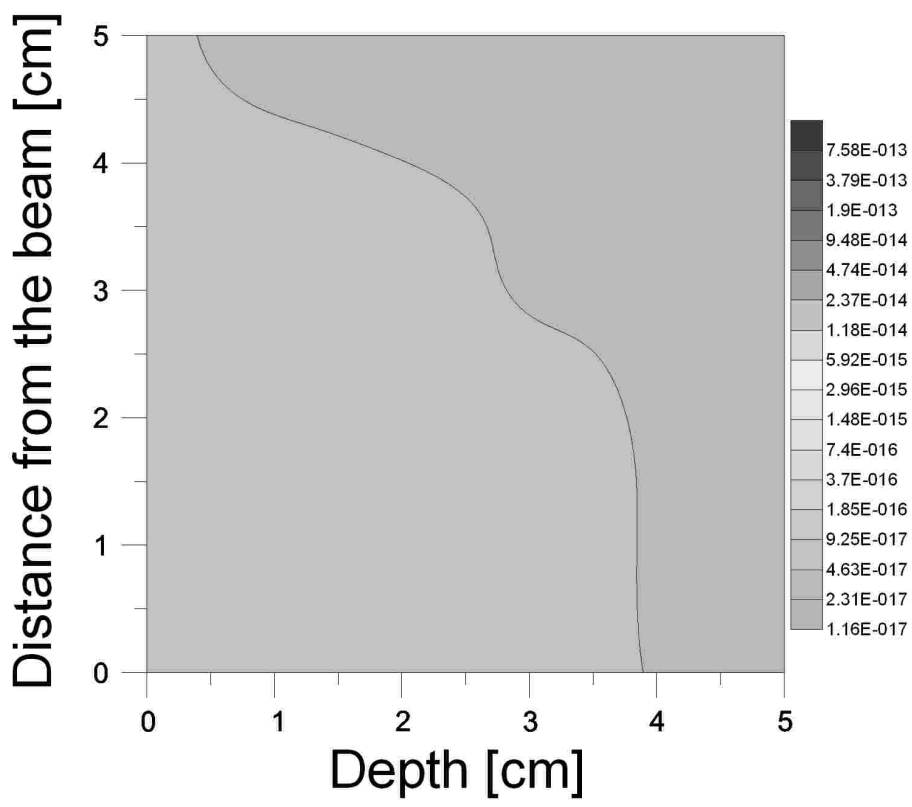

Figure 2b. The dependence of the fast neutron dose $[\mathrm{G} y / \mathrm{n}]$ on the depth and the distance from the beam for $1 \mathrm{keV}$ beam energy 


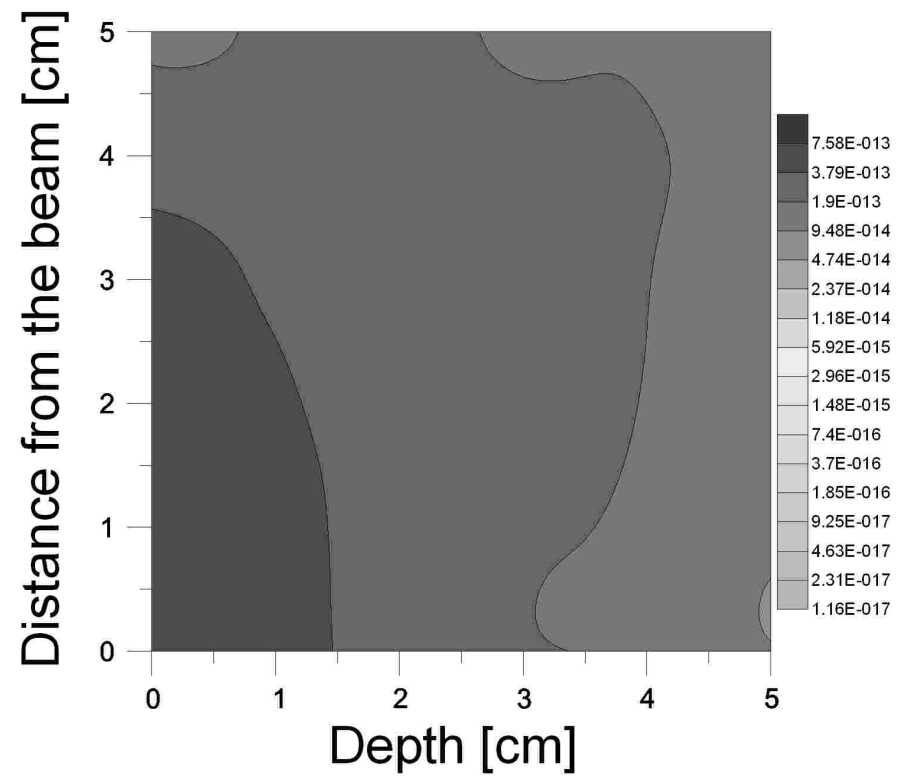

Figure 2c. The dependence of the fast neutron dose $[\mathrm{Gy} / \mathrm{n}]$ on the depth and the distance from the beam for $1 \mathrm{MeV}$ beam energy

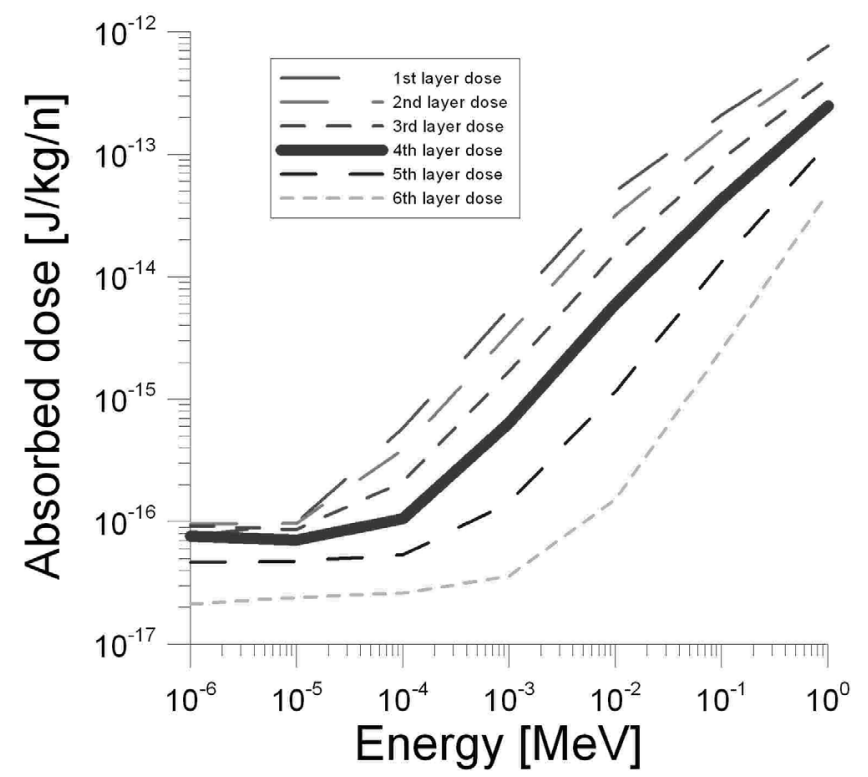

Figure 3. The dependence of the fast neutron dose on the beam energy for each particular layer. The thick line corresponds to the values obtained for the depth of $2-3 \mathrm{~cm}$, typical tumor situation (layer 4) 


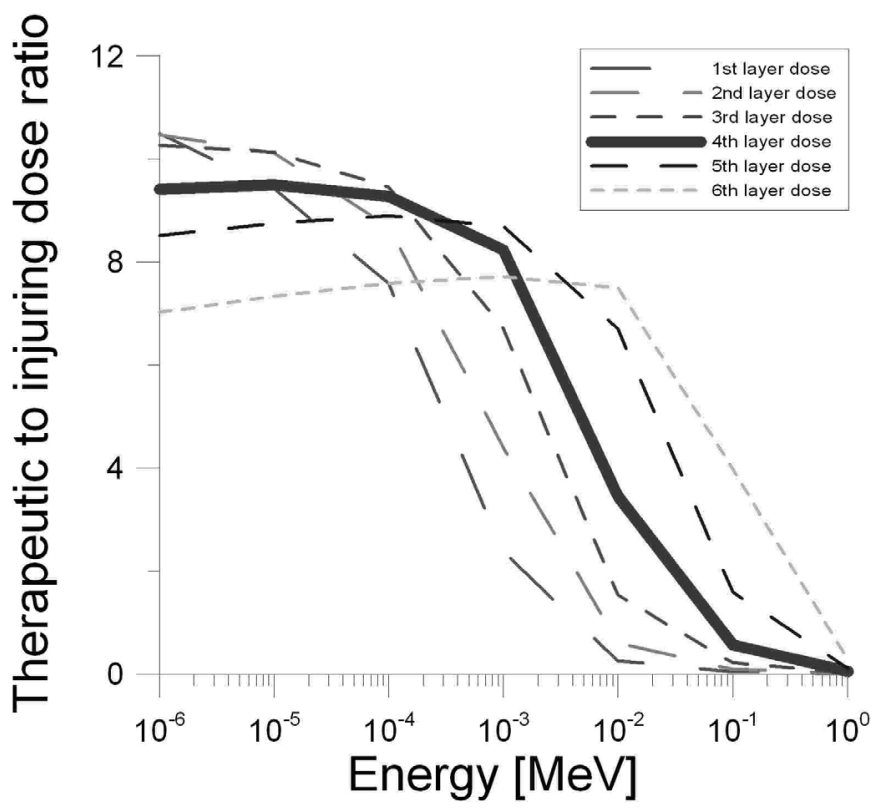

Figure 4. The ratio of the therapeutic to injuring dose for each particular layer. The thick line corresponds to the values obtained for the depth of $2-3 \mathrm{~cm}$, typical tumor situation (layer 4)

with the assumption that the proton is stopped within the cell where it was produced, so the kerma value equals the absorbed dose. The range of $1 \mathrm{MeV}$ protons in tissue is $2.27 \cdot 10^{-3} \mathrm{~g} / \mathrm{cm}^{2}$ [3], for the tissue density of $1.04 \mathrm{~g} / \mathrm{cm}^{3}$ it equals to about $2 \cdot 10^{-3} \mathrm{~cm}$ and it's two orders of magnitude smaller than the MCNP cell dimensions. The range for lower proton energies is even smaller, so such an assumption is justified.

Comparing to the distributions of the other dose components the spatial distribution is more flat when the dependence on the beam energy is very strong.

Figure 3 presents fast neutron dose for each particular layer as a function of the beam energy. For all the layers, the dose increases monotonically with the beam energy and the rise is higher for the outer layers. The energy dependence of the fast neutron dose component differs from the distributions for the boron and nitrogen components, therefore taking it into account changes the shape of the therapeutic to injuring ratio.

Figure 4 shows the ratio for each particular layer as a function of the beam energy. For low energies the ratio is very high and slumps with increasing energy. For the $4^{\text {th }}$ layer, corresponding to the typical tumor depth, the decrease is very rapid for energies above $1 \mathrm{keV}$. 


\section{Conclusions}

Taking into consideration the results obtained in our calculations we expect, that the best energy range for the boron neutron capture therapy is about $1 \mathrm{keV}$, due to the boron dose maximum in the most desired spot and the high therapeutic to injured ratio value. The result is in good agreement with the literature. The results will be used to design BNCT post at the Swierk reactor.

\section{Acknowledgements}

The work was supported by the Polish Ministry of Sciences and Higher Education, under grant no. PBZ/MEiN/01/2006/43.

\section{References}

[1] Tyminska K. Monte Carlo calculations of selected dose components in a head model for boron neutron capture therapy. Pol J Med Phys Eng. 2007; 13(3): 127-137.

[2] T-2 Nuclear Information Service [Internet]. Los Alamos: Los Alamos National Laboratory; c1998-2007. Total kerma coefficents, in units of $\mathrm{f} \mathrm{Gy} \mathrm{m} * * 2$, for various isotopes from thermal up to $150 \mathrm{MeV}$; [cited 2008]. Available from:

http:/t2.lanl.gov/data/TOTKERMA_neut

[3] Berger MJ, Coursey JS, Zucker MA, Chang J. Stopping-Power and Range Tables for Electrons, Protons, and Helium Ions [Internet]. Washington: U.S. Department of Commerce, Technology Administration, National Institute of Standards and Technology; 1993 Jan [updated: 2005 Aug; cited: 2008]. Available from: http://physics.nist.gov/PhysRefData/Star/Text/contents.html 